\title{
Elliptic operators on manifolds with singularities and $K$-homology
}

\author{
Anton Savin
}

August 4, 2018

\begin{abstract}
Elliptic operators on smooth compact manifolds are classified by K-homology. We prove that a similar classification is valid also for manifolds with simplest singularities: isolated conical points and edges. The main ingredients of the proof of these results are: Atiyah-Singer difference construction in the noncommutative case and Poincare isomorphism in K-theory for (our) singular manifolds. As an application we give a formula in topological terms for the obstruction to Fredholm problems on manifolds with edges.
\end{abstract}

MSC2000: 58J05(Primary) 19K33 35S35 47L15(Secondary)

\section{Introduction}

It is well known 1, 2, 3, that elliptic operators on a smooth closed manifold $M$ are classified by the $K$-homology group of the manifold. Namely, there is an isomorphism

$$
\operatorname{Ell}(M) \simeq K_{0}(M),
$$

where $\operatorname{Ell}(M)$ is the group of stable homotopy classes of elliptic pseudodifferential operators on the manifold, while $K_{0}(M)$ is the (even) $K$-homology group, considered as the generalized homology theory dual to Atiyah-Hirzebruch $K$-theory (see 4]).

Both sides of (11) are well-defined even if the manifold or the operators have singularities. More precisely, the right hand side is defined for finite complexes, while the left hand side can be defined at least for simplest singularity types: isolated points, edges, corners (see e.g. [5], 6], and also [7). It turns out that (10) holds for manifolds with singularities. As the main result of this paper, we prove that the isomorphism is valid for manifolds with simplest singularities.

Let us explain the scheme of obtaining the isomorphism. We start from the smooth case. Here the homotopy classification is given already by the Atiyah-Singer difference construction

$$
\chi: \operatorname{Ell}(M) \stackrel{\simeq}{\longrightarrow} K_{c}\left(T^{*} M\right) .
$$


Here $K_{c}$ denotes $K$-theory with compact support. Having in mind $K$-homological terminology, we note that we can apply Poincaré isomorphism in $K$-theory on the cotangent bundle to the latter group:

$$
p: K_{c}\left(T^{*} M\right) \stackrel{\simeq}{\longrightarrow} K_{0}(M)
$$

and obtain the desired isomorphism (11).

We now consider the case of singular $M$. Can one apply the same approach to prove the desired isomorphism? It turns out that this scheme can be applied modulo certain modifications.

Namely, the symbols of operators on singular manifolds define noncommutative algebras. Hence, it is natural to work in the $K$-theory of algebras. Then the topological $K$-group $K_{c}\left(T^{*} M\right)=K_{c}^{0}\left(T^{*} M\right)$ of the cotangent bundle in Eq. (2) is replaced by the $K_{0}$-group of a certain algebra (we denote it by $\mathcal{A}$ ) associated with the Calkin algebra of our operators and the algebra that defines the bundles, in sections of which our operators are acting. Hence, it remains to construct the isomorphism

$$
p: K_{0}(\mathcal{A}) \longrightarrow K_{0}(M)
$$

- an analog of Poincaré isomorphism (31), and we give here its construction on the example of simplest singularities like the conical point and the edge. Thus, we obtain the stable homotopy classification for elliptic operators on manifolds with such singularities.

The homotopy classification (11) has numerous applications. Let us now note one of them. In elliptic theory on manifolds with singularities, it is well known [5], 8, 9] that often an operator elliptic in the interior part of the manifold may not be Fredholm. Moreover, the Fredholm property can not be achieved even if we allow homotopies of the operator near the singularity. (This phenomenon goes back to the theory of boundary value problems, where there exists the Atiyah-Bott obstruction 10 to elliptic boundary conditions for elliptic operators on manifolds with boundary.) We will show that the homotopy classification enables one to write explicit formula for the obstruction. This formula gives the same result as in the previous computations in [10, 11].

Let us now briefly describe the contents of the paper. In the first section we give the abstract statement of the homotopy classification problem for elliptic operators. Then we formulate the main results of the paper - homotopy classifications for simplest classes of manifolds with singularities: conical points and edges. We would like to mention to simplify the presentation for manifolds with edges we use the algebra of pseudodifferential operators with discontinuous symbols introduced in [1]. The proofs of the classifications are given in the fourth section. They are based on the generalization (in Section 3) of the Atiyah-Singer difference construction 12 to the noncommutative case and Mayer-Vietoris arguments. Section 5 contains some applications of the homotopy classification. We conclude the paper with an appendix, where some basic facts about analytic $K$-homology are summarized.

Let us mention some papers related to the results we obtain. The homotopy classification for operators on manifolds with one singular point was obtained in 
[13. The Poincaré duality in terms of operator algebras on manifolds with isolated singularities was studied in a recent paper [14]. The classification of some operators on smooth manifolds with corners in terms of $K$-homology is obtained in [15]. Added in proof. The homotopy classification of edge-degenerate elliptic operators on manifolds with edges was obtained in [16] using the methods of the present paper.

The author is grateful to Prof. B. Yu. Sternin for a number of valuable remarks he made, while the paper was in preparation. The results of the paper were reported at the International Conference "Operator algebras on singular manifolds" Potsdam, Germany, March 2003, Workshop "Index problems" Paris, April 2004 and other meetings. The work was partially supported by RFBR grants NN 02-01-00118, 02-01-00928.

\section{Homotopy classification problem}

Abstract operators in subspaces. Consider a pair of algebras $\mathcal{A}_{0} \subset \mathcal{A}$ inside the algebra $\mathcal{B}(H)$ of bounded operators on a separable Hilbert space $H$. We will assume for simplicity that $\mathcal{A}$ contains the ideal of compact operators $\mathcal{K}=\mathcal{K}(H)$.

We call $\mathcal{A}$ the algebra of operators, its Calkin algebra $\mathcal{A} / \mathcal{K}$ - the algebra of symbols, while algebra $\mathcal{A}_{0}$ - algebra of functions. By $\operatorname{Im} A$ we denote the range of mapping $A$.

Definition 1 A linear operator

$$
D: \operatorname{Im} P \longrightarrow \operatorname{Im} Q
$$

is called operator in subspaces (see [17]) determined by the pair $P, Q \in \operatorname{Mat}\left(\mathcal{A}_{0}, n\right)$ of matrix $n$ by $n$ projections $\left(P^{2}=P, Q^{2}=Q\right)$ with entries in $\mathcal{A}_{0}$ and matrix operators $D \in \operatorname{Mat}(\mathcal{A}, n)$ satisfying the inclusion $D(\operatorname{Im} P) \subset \operatorname{Im} Q$ (algebraically this is equivalent to: $Q D P=D P)$.

Two operators in subspaces are equal, if they define the same restrictions (5).

This definition is an abstract analog of the notion of operator acting in sections of vector bundles.

Example 1 Consider the pair $\mathcal{A}_{0}=C^{\infty}(M), \mathcal{A}=\Psi(M)$, where $\Psi(M)$ denotes the algebra of pseudodifferential operators of order zero on a closed smooth manifold $M$. Both algebras are considered as subalgebras of operators acting on $L^{2}(M)$. In this case, projections with entries in $C^{\infty}(M)$ define sections of vector bundles over $M$, while the operator in subspaces (5) is merely an operator acting in vector bundle sections.

Definition 2 Operator $D: \operatorname{Im} P \rightarrow \operatorname{Im} Q$ is elliptic, if there exists an almostinverse operator, i.e. operator $D^{\prime}: \operatorname{Im} Q \rightarrow \operatorname{Im} P, D^{\prime} \in \operatorname{Mat}(\mathcal{A}, n)$ such that the compositions $D D^{\prime}$ and $D^{\prime} D$ give (modulo compact operators) identity mappings in subspaces $\operatorname{Im} Q$ and $\operatorname{Im} P$. 
Stable homotopies. Group $\operatorname{Ell}\left(\mathcal{A}_{0}, \mathcal{A}\right)$. Two elliptic operators $\left(D_{0}, P_{0}, Q_{0}\right)$ and $\left(D_{1}, P_{1}, Q_{1}\right)$ are homotopic, if they can be connected by a continuous family of elliptic operators: ${ }^{1}$

$$
D_{t}: \operatorname{Im} P_{t} \rightarrow \operatorname{Im} Q_{t}, \quad t \in[0,1], \quad P_{t}, Q_{t} \in \operatorname{Mat}\left(\mathcal{A}_{0}, n\right),
$$

Definition 3 Two elliptic operators are stably homotopic, if they become homotopic after addition of some trivial operators to each of them. Here trivial operators are identity operators of arbitrary matrix order (i.e., triples $(1, P, P)$ with an arbitrary projection $P$ ).

It is easy to check that stable homotopy is an equivalence relation. $\operatorname{By} \operatorname{Ell}\left(\mathcal{A}_{0}, \mathcal{A}\right)$ we denote the set of elliptic operators, modulo stable homotopies. This set is an Abelian group with respect to the direct sum of operators in subspaces. The inverse element is given by the almost-inverse operator and the zero is represented by trivial operators.

The main problem we consider in this paper is the problem of computing the group Ell for some particular classes of algebras. We refer to this problem as the homotopy classification problem for elliptic operators generated by the pair $\left(\mathcal{A}_{0}, \mathcal{A}\right)$

Symbols. Similar to operators one can introduce the notion of symbol in subspaces

$$
(\sigma, P, Q) \text {. }
$$

Here $P, Q$ are projections in matrix algebras over $\mathcal{A}_{0}$ as above, while the matrix operator $\sigma \in \operatorname{Mat}(\mathcal{A} / \mathcal{K}, n)$ has components in the Calkin algebra. As usual it is easy to prove that the symbol allows one to reconstruct the operator uniquely up to compact operators, and the existence of a homotopy between two operators is equivalent to the existence of homotopies of their symbols. Therefore, classification of elliptic operators up to stable homotopy is equivalent to a similar classification of elliptic symbols. The latter is technically simpler.

\section{Main results}

\subsection{Operators on manifolds with conical points}

Manifolds. The simplest manifold with singularities is the manifold $\mathcal{M}$ with a conical point. It can be obtained if we identify all the boundary points of a closed compact smooth manifold $M$ with nonempty boundary $\partial M$

$$
\mathcal{M}=M / \partial M
$$

\footnotetext{
${ }^{1}$ Here an operator family $D_{t}: \operatorname{Im} P_{t} \rightarrow \operatorname{Im} Q_{t}$ (the source and the target spaces depend on $t$ ) is continuous if the projections defining the spaces vary continuously and the family $D_{t}$ is a restriction of some continuous family $\widetilde{D}_{t} \in \operatorname{Mat}(\mathcal{A}, n)$.
} 
This space has only one point at which it is not smooth. This is the point, to which the whole initial boundary is collapsed. Near the singularity the space looks like a neighborhood of the vertex of the cone with base $\partial M$

$$
K_{\partial M}=\partial M \times \overline{\mathbb{R}}_{+} / \partial M \times\{0\} .
$$

Algebra of operators. On $\mathcal{M}$, we consider the algebra $\Psi(\mathcal{M})$ of cone pseudodifferential operators of order zero (e.g., see [18, 19])

$$
D: L^{2}(\mathcal{M}) \longrightarrow L^{2}(\mathcal{M})
$$

acting in weighted Sobolev space of weight and order zero. These spaces will be denoted by $L^{2}(\mathcal{M})$.

Let us recall that the Calkin algebra ${ }^{2} \Psi(\mathcal{M}) / \mathcal{K}$ consists of compatible pairs

$$
\Psi(\mathcal{M}) / \mathcal{K}=\left\{\left(\sigma, \sigma_{c}\right) \in C^{\infty}\left(S^{*} M\right) \oplus \Psi_{p}(\partial M)|\sigma|_{\partial S^{*} M}=\operatorname{smbl} \sigma_{c}\right\}
$$

- the first component $\sigma(D)$ is the principal symbol - function on the cosphere bundle $S^{*} M$ of the manifold, smooth up to the boundary $\partial S^{*} M$;

- the second component $\sigma_{c}(D)(p)$ - the conormal symbol, is a parameterdependent family with $p \in \mathbb{R}$ (see [20]) of zero order pseudodifferential operators on the boundary $\partial M$. The algebra of parameter-dependent families is denoted by $\Psi_{p}(\partial M)$.

The compatibility condition in (7) involves the symbol mapping for parameterdependent families:

$$
\operatorname{smbl}: \Psi_{p}(\partial M) \longrightarrow C^{\infty}\left(S\left(T^{*} \partial M \times \mathbb{R}\right)\right) .
$$

Finally, we assume in (17) that an identification $\left.S^{*} M\right|_{\partial M}=S\left(T^{*} \partial M \times \mathbb{R}\right)$ is chosen.

We refer the reader to the cited papers for explicit formulas which allow one to compute both symbols $\sigma(D), \sigma_{c}(D)$ for a given operator $D$ and, conversely, to construct an operator starting from a compatible pair of symbols $\left(\sigma, \sigma_{c}\right)$.

Homotopy classification. Denote by $\operatorname{Ell}(\mathcal{M})$ the group of stable homotopy classes of elliptic operators associated with the inclusion $C^{\infty}(M) \subset \Psi(\mathcal{M})$ :

$$
\operatorname{Ell}(\mathcal{M})=\operatorname{Ell}\left(C^{\infty}(M), \Psi(\mathcal{M})\right) .
$$

In terms of elliptic operators one can say that this group is generated by elliptic operators

$$
D: L^{2}(\mathcal{M}, E) \longrightarrow L^{2}(\mathcal{M}, F),
$$

acting on sections of arbitrary vector bundles $E, F \in \operatorname{Vect}(M)$.

The above description of the Calkin algebra $\Psi(\mathcal{M}) / \mathcal{K}$ shows that the commutator $[D, f]$ with function $f \in C^{\infty}(M)$ is compact if the function is constant on

\footnotetext{
${ }^{2}$ We shall assume that the algebras of pseudodifferential operators contain the ideal of compact operators.
} 
the boundary. By continuity this gives the compactness of the commutator for an arbitrary continuous function on the (compact) singular space $\mathcal{M}$

$$
[D, f] \in \mathcal{K}, \quad \text { for all } f \in C(\mathcal{M})
$$

It follows (see Section 6) that an arbitrary elliptic operator $D$ defines an element in analytic $K$-homology of the singular space $\mathcal{M}$ (such elements were first defined in 21]). We denote this element by

$$
\chi(D) \in K_{0}(\mathcal{M}) .
$$

Theorem 1 The mapping $D \mapsto \chi(D)$ defines an isomorphism of groups:

$$
\operatorname{Ell}(\mathcal{M}) \stackrel{\chi}{\simeq} K_{0}(\mathcal{M})
$$

Manifolds with several conical points. Similarly, one can consider manifold $\mathcal{M}$ with $N>1$ distinct conical points. In other words, when the singular space is obtained from a manifold with boundary such that the connected components of the boundary are arranged into $N$ nonintersecting groups:

$$
\partial M=\Omega_{1} \sqcup \Omega_{2} \ldots \sqcup \Omega_{N},
$$

and each group $\Omega_{i}$ is then collapsed to a point $p t_{i}$. Analytically, this means that we consider operators with $N$ conormal symbols. The isomorphism of Theorem 1 remains true in this case.

Remark 1 The homotopy classification in the case of one singular point was obtained in [13. It was shown that the $\operatorname{group} \operatorname{Ell}(\mathcal{M})$ is isomorphic to the direct sum $K_{c}^{0}\left(T^{*} M\right) \oplus \mathbb{Z}$. Theorem 1 gives the same answer in this case. Indeed:

$$
K_{0}(\mathcal{M})=K_{0}(M / \partial M) \simeq K_{0}(M, \partial M) \oplus \mathbb{Z} \simeq K_{c}^{0}\left(T^{*} M\right) \oplus \mathbb{Z} .
$$

Here we use Poincaré isomorphism on manifolds with boundary [2]

\subsection{Operators with discontinuous symbols in fiber bun- dles}

In this subsection we study the classification problem for a class of elliptic operators on fibered manifolds. These operators will be used in the next subsection to obtain the homotopy classification on manifolds with fibered boundary.

Operators on a fibered manifold. Consider a smooth locally trivial fiber bundle

$$
\pi: Y \rightarrow X
$$

Assume that the base $X$ and the fiber (denoted by $\Omega$ ) are compact closed manifolds.

Consider the algebra generated by pseudodifferential operators of order zero on $Y$ and families of pseudodifferential operators of order zero acting in the fibers, see [11. Denote this algebra by $\Psi(Y, \pi)$. Its elements can be considered also as 
operators on $Y$ with symbols having discontinuities of a special form that we now describe. (Symbols discontinuous in covariables were studied earlier in [22].)

Denote by $\overline{S^{*} Y \backslash \pi^{*} S^{*} X}$ the compactification of the complement $S^{*} Y \backslash \pi^{*} S^{*} X$, for which a sequence $\left(x_{i}, \omega_{i}, \xi_{i}, \eta_{i}\right)$ converges to a point in $\pi^{*} S^{*} X$, if in addition to the usual convergence one also has the convergence for the quotients $\eta_{i} /\left|\eta_{i}\right|$ (here $x, \omega$ are the coordinates along the base and the fiber, while $\xi, \eta-$ are the dual coordinates). It is easy to show that $\overline{S^{*} Y \backslash \pi^{*} S^{*} X}$ is a manifold with boundary. ${ }^{3}$ This boundary is diffeomorphic to the complement $S^{*} Y \backslash U_{\pi^{*} S^{*} X}$ to a collar neighborhood $U_{\pi^{*} S^{*} X}$ of the submanifold $\pi^{*} S^{*} X$.

Now the Calkin algebra of $\Psi(Y, \pi)$ can be described (see [1]) as the subalgebra in the direct sum of algebras:

$$
\Psi(Y, \pi) / \mathcal{K} \subset C^{\infty}\left(\overline{S^{*} Y \backslash \pi^{*} S^{*} X}\right) \oplus C^{\infty}\left(S^{*} X, \Psi(\Omega)\right),
$$

determined by the compatibility condition:

$$
\Psi(Y, \pi) / \mathcal{K}=\left\{(\sigma, \widehat{\sigma}) \quad|\sigma|_{\partial S^{*} Y \backslash \pi^{*} S^{*} X}=\operatorname{smbl} \widehat{\sigma}\right\} .
$$

For an operator $A \in \Psi(Y, \pi)$ the first component $\sigma(A)$ is the principal symbol of the operator. The principal symbol is a function on $S^{*} M$ smooth except $\pi^{*} S^{*} X$. The second component $\widehat{\sigma}(A)$ - is called the operator symbol. The operator symbol is a function on the cosphere bundle of the base with values in the algebras $\Psi(\Omega)$ of pseudodifferential operators in the fibers.

Consider the pair of algebras $C^{\infty}(X, \Psi(\Omega)) \subset \Psi(Y, \pi)$. The corresponding group of stable homotopy classes of elliptic operators is denoted by $\operatorname{Ell}(Y, \pi)$. It is generated by operators

$$
D: \operatorname{Im} P_{1} \longrightarrow \operatorname{Im} P_{2},
$$

acting on the fibered manifold, where the projections $P_{1,2}: L^{2}\left(Y, E_{1,2}\right) \rightarrow L^{2}\left(Y, E_{1,2}\right)$ are induced by continuous families of pseudodifferential operators in the fibers. We refer the reader to [11] for a detailed study of these operators, in particular, for the proof of (9).

Homotopy classification. The properties of the Calkin algebra $\Psi(Y, \pi) / \mathcal{K}$ show that for an elliptic operator $D \in \operatorname{Ell}(Y, \pi)$ the commutator $[D, f]$ is compact provided $f$ is constant in the fibers of $\pi$. Therefore, operator $D$ defines an element

$$
\chi(D) \in K_{0}(X)
$$

in analytic $K$-homology of the base.

Theorem 2 One has an isomorphism:

$$
\operatorname{Ell}(Y, \pi) \stackrel{\chi}{\simeq} K_{0}(X) .
$$

\footnotetext{
${ }^{3}$ The boundary fibers over $Y$, with fiber over a point $(x, \omega)$ equal to the product of the cospheres $S_{x}^{*} X \times S_{\omega}^{*} \Omega$.
} 
Remark 2 A similar result holds in the case, when the base of the fiber bundle has a nonempty boundary. In this case we consider the algebra generated by the operators which near the boundary act as operators of multiplication by functions $f \in C^{\infty}(Y)$. The corresponding group $\operatorname{Ell}(Y, \pi)$ will be isomorphic in this case to the group $K_{c}^{0}\left(T^{*} \stackrel{\circ}{X}\right) \simeq K_{0}(X)$, where $\stackrel{\circ}{X}$ denotes the interior part. The same classification can be obtained if one considers more general operators, which near the boundary are defined by operator-valued functions $f \in C^{\infty}(X, \Psi(\Omega))$. This result can be obtained by the same method.

\subsection{Operators on manifolds with fibered boundary}

Manifolds with fibered boundary. Let $M$ be a compact manifold with boundary, and the boundary is the total space of a locally-trivial fiber bundle with fiber $\Omega$. We will fix some extension of the fibration to some collar neighborhood of the boundary $U_{\partial M} \simeq \partial M \times[0,1)$ and denote the extended projection by

$$
\pi: \partial M \times[0,1] \rightarrow X \times[0,1] .
$$

Let $\mathcal{M}$ be the manifold with singularities, obtained from $M$ by the identification of points in the fibers of $\pi$. A homotopy equivalent space can be obtained if we identify only the points in the fibers at the boundary $\partial M$. This space is referred to as a manifold with edge $X$.

The aim of the present subsection is to describe an elliptic theory, whose homotopy classification produces the group $K_{0}(\mathcal{M})$.

Operator algebra. Denote by $\Psi(M, \pi) \subset \mathcal{B}\left(L^{2}(M)\right)$ the algebra generated by:

- the usual pseudodifferential operators of order zero over the interior part of the manifold;

- in a neighborhood $\partial M \times[0,1)$ of the boundary by operators from the algebra $\Psi(\partial M \times[0,1), \pi)$ (see Remark 2 of the previous section);

- compact operators.

In other words, the operators from the algebra $\Psi(M, \pi)$ are the usual pseudodifferential operators far from the boundary, and in a neighborhood of the boundary are operators corresponding to the fibration $\pi$.

Homotopy classification. Let $\operatorname{Ell}(M, \pi)$ be the group of stable homotopy classes of elliptic operators corresponding to the embedding of algebras $C^{\infty}(M) \subset \Psi(M, \pi)$.

The symbolic calculus of the usual pseudodifferential operators and operators in the fibration $\pi$ show that for an operator $D \in \Psi(M, \pi)$, and $f \in C^{\infty}(M)$ the commutator $[D, f]$ is compact, if the function is constant in the fibers of $\pi$, i.e., it is induced by a continuous function on the manifold with edge. Hence (see Section 6), an elliptic operator $D$ from $\operatorname{Ell}(M, \pi)$ defines an element in $K$-homology of the singular space $\mathcal{M}$. Denote this element as

$$
\chi(D) \in K_{0}(\mathcal{M}) .
$$


Theorem 3 One has

$$
\operatorname{Ell}(M, \pi) \stackrel{\chi}{\simeq} K_{0}(\mathcal{M})
$$

\section{$3 \quad$ Ell-theory and $K$-theory}

\subsection{Difference construction}

The aim of this subsection is to prove that the $\operatorname{group} \operatorname{Ell}\left(\mathcal{A}_{0}, \mathcal{A}\right)$ is isomorphic to the $K$-group of some algebra constructed from the pair $\left(\mathcal{A}_{0}, \mathcal{A}\right)$. The isomorphism generalizes the Atiyah-Singer difference construction [12].

In addition to the conditions of Section 1, we will assume that $\mathcal{A}$ and $\mathcal{A}_{0}$ are $C^{*}$-algebras. For definiteness we will assume that $\mathcal{A}_{0}$ is unital.

By $\mathrm{Con}_{f}$ denote the cone of a monomorphism $f: A_{0} \rightarrow A$. We will also write $\operatorname{Con}\left(A_{0}, A\right)$, when $f$ is the inclusion of a subalgebra. Recall that the cone is defined as the subalgebra of the direct sum $A_{0} \oplus C_{0}([0,1), A)$ :

$$
\mathrm{Con}_{f}=\left\{\left(a_{0}, a(t)\right) \in A_{0} \oplus C_{0}([0,1), A) \mid f\left(a_{0}\right)=a(0)\right\} .
$$

Difference element of an elliptic symbol. Let us prove that the symbol of an elliptic operator in subspaces

$$
D: \operatorname{Im} P \rightarrow \operatorname{Im} Q
$$

defines an element in the $K$-group $K_{0}\left(\operatorname{Con}\left(\mathcal{A}_{0}, \mathcal{A} / \mathcal{K}\right)\right)$ of the cone of the natural mapping $\mathcal{A}_{0} \rightarrow \mathcal{A} / \mathcal{K}$. This element is constructed as follows.

To shorten the notation, we denote the algebra $\operatorname{Con}\left(\mathcal{A}_{0}, \mathcal{A} / \mathcal{K}\right)$ by $\operatorname{Con}$, while the algebra with adjoined unit by $\mathrm{Con}^{+}$. The latter algebra also consists of pairs $(a, a(t))$. The only difference is that for $\mathrm{Con}^{+}$the function $a(t)$ at $t=1$ can be an arbitrary scalar:

$$
a(1)=\lambda I d, \quad \lambda \in \mathbb{C} .
$$

Let us define a matrix projection $\mathcal{P}$ with entries in $\mathrm{Con}^{+}$:

$$
\mathcal{P}=\left(P, P_{t}\right) \in \operatorname{Mat}\left(\mathrm{Con}^{+}, N\right) .
$$

The second term in this pair $P_{t} \in \operatorname{Mat}(\mathcal{A} / \mathcal{K}, N)$ is a family of projections defined for $t \in[0,1]$ as

$$
P_{t}=P \cos ^{2} \varphi+Q \sin ^{2} \varphi+\cos \varphi \sin \varphi\left(\sigma P+\sigma^{-1} Q\right), \quad \varphi=\frac{\pi}{2} t
$$

(here $\sigma \in A$ denotes the symbol of $D, \sigma^{-1}$ is the symbol of the almost-inverse operator). The homotopy of projections (14) is called rotation homotopy. ${ }^{4}$ The homotopy is well defined (i.e., for all $t$ the operator $P_{t}$ is a projection), provided the

\footnotetext{
${ }^{4}$ The term rotation is motivated by the fact that in the case of orthogonal projections $P, Q$ and an isometric isomorphism $\sigma$ the vectors in the range of $P_{t}$ are obtained as the rotation by angle $\pi t / 2$ of $v \in \operatorname{Im} P$ towards $\sigma v \in \operatorname{Im} Q$.
} 
ranges of $P$ and $Q$ are orthogonal. The orthogonality is valid, if, for instance, the projections have the following block-diagonal form

$$
P=\left(\begin{array}{cc}
* & 0 \\
0 & 0
\end{array}\right), \quad Q=\left(\begin{array}{cc}
0 & 0 \\
0 & I d_{n}
\end{array}\right), \quad P, Q \in \operatorname{Mat}\left(\mathcal{A}_{0}, m+n\right)
$$

(an arbitrary pair $(P, Q)$ can be reduced to this form by stable homotopies).

Let $\mathcal{P}_{0} \in \operatorname{Mat}\left(\mathrm{Con}^{+}, m+n\right)$ be the projection with components $(Q, Q)$ (cf. (13) $)$. The element

$$
\chi(\sigma(D))=[\mathcal{P}]-\left[\mathcal{P}_{0}\right] \in K_{0}(\text { Con }),
$$

is called the difference element of the elliptic operator $D$.

\section{Difference construction}

Theorem 4 The mapping $D \mapsto \chi(\sigma(D))$ induces a group isomorphism (difference construction)

$$
\operatorname{Ell}\left(\mathcal{A}_{0}, \mathcal{A}\right) \stackrel{\chi}{\simeq} K_{0}\left(\operatorname{Con}\left(\mathcal{A}_{0}, \mathcal{A} / \mathcal{K}\right)\right)
$$

Example 2 On a smooth manifold, the Calkin algebra $\overline{\Psi(M)} / \mathcal{K}$ (here $\overline{\Psi(M)}$ is the norm closure in $\left.\mathcal{B}\left(L^{2}(M)\right)\right)$ is isomorphic to the algebra $C\left(S^{*} M\right)$ of continuous functions on the cosphere bundle of the manifold, the cone of the embedding $C(M) \subset C\left(S^{*} M\right)$ is isomorphic to the algebra $C_{0}\left(T^{*} M\right)$ of functions on the cotangent bundle vanishing at infinity. In this case the theorem gives the Atiyah-Singer isomorphism: the group of stable homotopy classes of elliptic pseudodifferential operators on $M$ is isomorphic to the $K$-group $K_{c}^{0}\left(T^{*} M\right)$.

Proof. Let us prove that the difference construction is well defined. Actually, this follows from the definition of the groups Ell and $K_{0}$. Indeed, the difference element of a trivial operator is zero in the $K$-group, while a homotopy of elliptic operators induces a homotopy of the corresponding projections $\mathcal{P}$ and $\mathcal{P}_{0}$ in (15).

The invertibility of the difference homomorphism is proved by first constructing the left inverse mapping to $\chi$ and then proving the surjectivity of $\chi$. These two facts prove the desired isomorphism. Let us prove these facts.

1. Let us construct the inverse mapping

$$
\chi^{-1}: K_{0}(\mathrm{Con}) \rightarrow \operatorname{Ell}\left(\mathcal{A}_{0}, \mathcal{A}\right)
$$

Consider an element $[\mathcal{P}]-[\mathcal{Q}] \in K_{0}($ Con $)$, where $\mathcal{P}, \mathcal{Q}$ are projections in matrix algebras over $\mathrm{Con}^{+}$. We can assume that $\mathcal{Q}=I d_{n} \oplus 0_{m}$. Then for the projection $\mathcal{P}=\left(P, P_{t}\right)$ we define

$$
\chi^{-1}([\mathcal{P}]-[\mathcal{Q}]) \stackrel{\text { def }}{=}\left[U: \operatorname{Im} P \longrightarrow \operatorname{Im} P_{1}\right],
$$

where the symbol of an elliptic operator $U$ is defined as the value at $t=1$ of the solution of the Cauchy problem:

$$
\left\{\begin{array}{c}
\dot{u}_{t}=\left[\dot{P}_{t}, P_{t}\right] u_{t} \\
u_{0}=1
\end{array}\right.
$$


This procedure requires the smoothness in $t$ of the homotopy. Nonetheless, this is sufficient to define the mapping (17). This follows from the fact that the subalgebra in Con, corresponding to smooth families of projections $P_{t}$, is local (in the sense of [23]) and, thus, defines the same $K$-group as the algebra Con.

2. The equality $\chi^{-1} \circ \chi=I d$ is obtained by a direct computation, since (18) can be solved explicitly for the projection defined by (14). The details of the computation are left to the reader.

3. To prove the surjectivity of $\chi$ one has to show that an arbitrary element

$$
[\mathcal{P}]-[\mathcal{Q}] \in K_{0}(\mathrm{Con})
$$

can be represented in the form (15), for a rotation homotopy (14) defined by some elliptic symbol $\sigma$.

Let us deform the pair $\mathcal{P}, \mathcal{Q}$ to the desired form. To this end, it is enough to assume that the second projection is trivial: $\mathcal{Q}=(Q, Q)$, as in (15). In this case $Q$ is determined by $\mathcal{P}=\left(P, P_{t}\right)$, or, more precisely, by the value of its second component at $t=1$. Hence, it will be enough to construct only homotopies of $\mathcal{P}$, without mentioning explicitly the homotopy of $\mathcal{Q}$.

We construct the desired homotopy $\mathcal{P}=\left(P, P_{t}\right)$ in two steps.

First, we deform the homotopy to obtain a homotopy $P_{t}$, which for $t \in[1 / 2,1]$ is the rotation homotopy from $P_{1 / 2}$ towards $P_{1}$, while the projections $P_{t}$ for all $t \leq 1 / 2$ are orthogonal to $P_{1}$. This can be accomplished by a stabilization (passing to matrices twice bigger as the original ones) and by superposition of the original homotopy with the rotation homotopy, which connects the projections $P_{1} \oplus 0$ and $0 \oplus P_{1}$.

Second, we extend the rotation homotopy obtained previously from the halfinterval $[1 / 2,1]$ to the whole $[0,1]$. To this end we deform the homotopy of projections $P_{t}$ with deformation parameter $\varepsilon \in[0,1 / 2]$ according to the formula

$$
P_{\varepsilon, t}=\left\{\begin{array}{cc}
P_{t}, & \text { for } t \leq \varepsilon, \\
\text { rotation by angle } \frac{(t-\varepsilon) \pi}{(1-\varepsilon) 2} & \text { for } t \geq \varepsilon, \\
\text { from } P_{\varepsilon} \text { towards } P_{1}, &
\end{array}\right.
$$

where in the second case the rotation between the projections with a given homotopy between them is defined by substituting in (14) the solution of the Cauchy problem (18). By construction, for $\varepsilon=0$ we obtain the rotation homotopy between $P_{0}$ and $P_{1}$. Therefore, the initial element (19) is indeed a difference element for some elliptic operator.

This proves that the difference construction is surjective.

Remark 3 (families of operators) One can generalize the difference construction to the case of continuous families of elliptic operators. This generalization is done by standard techniques. Since this will be used later (see Remark 4), we will formulate the result: families of elliptic operators corresponding to a pair $\left(\mathcal{A}_{0}, \mathcal{A}\right)$ and parametrized by a compact space $X$ are classified by the $K$-group of the algebra $C(X$, Con $)$ of continuous Con-valued functions on $X$. 
Remark 4 Operator families can be used to define the odd elliptic group corresponding to the group $K_{1}$ of the mapping cone. Namely, let $\operatorname{Ell}_{1}\left(\mathcal{A}_{0}, \mathcal{A}\right)$ be the group of stable homotopy classes of elliptic families $\left\{D_{\varphi}\right\}_{\varphi \in \mathbb{S}^{1}}$ such that for $\varphi=0$ $D_{\varphi}$ is trivial (i.e., the operator at $\varphi=0$ has components in the algebra $\mathcal{A}_{0}$ ). A family is trivial, if $D_{\varphi}$ is trivial for all $\varphi$. It is easy to express the odd groups in terms of the even ones:

$$
\operatorname{Ell}_{1}\left(\mathcal{A}_{0}, \mathcal{A}\right) \simeq \operatorname{Ell}\left(\Sigma \mathcal{A}_{0}, \Sigma \mathcal{A}\right)
$$

(Here $\Sigma A$ is the suspension $C_{0}((0,1), A)$ of $A$.) Hence, applying Theorem 4 we get the desired isomorphism

$$
\operatorname{Ell}_{1}\left(\mathcal{A}_{0}, \mathcal{A}\right) \simeq K_{1}\left(\operatorname{Con}\left(\mathcal{A}_{0}, \mathcal{A} / \mathcal{K}\right)\right)
$$

Here we use the suspension isomorphism.

\subsection{Mapping cone $K$-theory exact sequence}

One has an exact sequence

$$
\ldots \rightarrow K_{1}(\mathcal{A} / \mathcal{K}) \rightarrow K_{0}(\mathrm{Con}) \rightarrow K_{0}\left(\mathcal{A}_{0}\right) \longrightarrow K_{0}(\mathcal{A} / \mathcal{K}) \rightarrow K_{1}(\text { Con }) \ldots
$$

induced by the short exact sequence of algebras

$$
0 \rightarrow \Sigma(\mathcal{A} / \mathcal{K}) \longrightarrow \mathrm{Con} \longrightarrow \mathcal{A}_{0} \rightarrow 0 \text {. }
$$

The elements in (20) admit natural descriptions in terms of elliptic operators:

- the group $K_{1}(\mathcal{A} / \mathcal{K})$ corresponds to the subclass of matrix elliptic operators (i.e., the projections $P, Q$ are identity maps) and is formed by the stable homotopy classes of such operators;

- the mapping $K_{1}(\mathcal{A} / \mathcal{K}) \rightarrow K_{0}$ (Con) in (20) is induced by the embedding of matrix operators in the class of operators in arbitrary subspaces (i.e., subspaces defined by possibly nontrivial projections);

- the mapping $K_{0}(\mathrm{Con}) \rightarrow K_{0}\left(\mathcal{A}_{0}\right)$ takes an operator in subspaces to the difference of its projections;

- the boundary mappings $K_{*}\left(\mathcal{A}_{0}\right) \longrightarrow K_{*}(\mathcal{A} / \mathcal{K})$ are induced by the algebra homomorphism $f: \mathcal{A}_{0} \rightarrow \mathcal{A} / \mathcal{K}$ (e.g., see [24]);

- the mapping $K_{0}(\mathcal{A} / \mathcal{K}) \rightarrow K_{1}(\mathrm{Con})$ takes a projection $P \in \operatorname{Mat}(\mathcal{A} / \mathcal{K}, n)$ to the family $P z+I d_{n}-P$, where $z=e^{i \varphi}$ is the coordinate on the circle.

\section{Proofs of the homotopy classifications}

In this section we give the proofs of the theorems stated in Section 2 , 


\subsection{Isolated singularities}

In this subsection we give the proof of Theorem 1 on the homotopy classification of elliptic operators on a manifold with a conical point.

1. Instead of proving the classification of elliptic operators, we shall classify elliptic symbols (according to Section 1 the two problems are equivalent).

2. Furthermore, the classification corresponding to the pair of algebras $C^{\infty}(M) \subset$ $\Psi(\mathcal{M})$ coincides with the classification for the closure of this pair with respect to the operator norm: $C(M) \subset \overline{\Psi(\mathcal{M})}$. Let us note that similarly to the original algebra, the Calkin algebra of the closure consists of compatible pairs

$$
\overline{\Psi(\mathcal{M})} / \mathcal{K}=\left\{\left(\sigma, \sigma_{c}\right) \in C\left(S^{*} M\right) \oplus \overline{\Psi_{p}(\partial M)}|\sigma|_{\partial S^{*} M}=\operatorname{smbl} \sigma_{c}\right\} .
$$

Here the closure $\overline{\Psi_{p}(\Omega)}$ of the algebra of parameter-dependent families is considered with respect to the norm

$$
\sup _{p \in \mathbb{R}}\left\|\sigma_{c}(p)\right\|_{L^{2}(\partial M) \rightarrow L^{2}(\partial M)} .
$$

This description follows from the well-known estimates of the norm for operators $D \in \Psi(\mathcal{M})$ modulo sums with compact operators:

$$
\inf _{K \in \mathcal{K}}\|D+K\|_{L^{2}(\mathcal{M}) \rightarrow L^{2}(\mathcal{M})}=\max \left(\max _{(x, \xi) \in S^{*} M}|\sigma(D)(x, \xi)|, \sup _{p \in \mathbb{R}}\left\|\sigma_{c}(D)(p)\right\|\right) .
$$

3. We can apply the difference construction of the previous section (Theorem 4) to the embedding of $C^{*}$-algebras $f: C(M) \rightarrow \overline{\Psi(\mathcal{M})} / \mathcal{K}$ :

$$
\operatorname{Ell}(\mathcal{M}) \simeq K_{0}\left(\operatorname{Con}_{f}\right)
$$

Thus, we will prove the isomorphism of the latter group and the $K$-homology group of the singular manifold.

4. Let us embed $\chi: K_{0}\left(\mathrm{Con}_{f}\right) \rightarrow K_{0}(\mathcal{M})$ in the diagram:

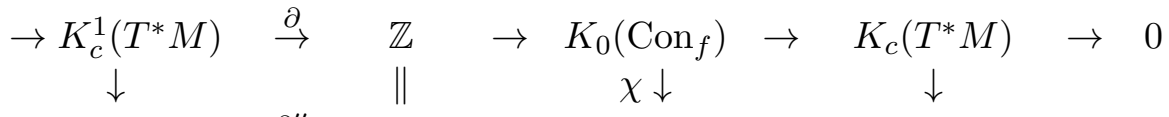

$$
\begin{aligned}
& \rightarrow K_{1}(M, \partial M) \stackrel{\partial^{\prime \prime}}{\rightarrow} K_{0}(p t) \rightarrow K_{0}(\mathcal{M}) \rightarrow K_{0}(M, \partial M) \rightarrow 0 .
\end{aligned}
$$

The bottom row of the diagram is the $K$-homology exact sequence for the pair $p t \subset \mathcal{M}$, while the upper row is the $K$-theory exact sequence for the ideal

$$
I \subset \operatorname{Con}_{f}
$$

of elements with zero principal symbol. For this ideal it is easy to obtain an isomorphism $I \simeq C_{0}((0,1) \times \mathbb{R}, \mathcal{K})$, and for the quotient we have

$$
\mathrm{Con}_{f} / I \simeq C_{0}\left(B^{*} M \backslash S^{*} M\right) \simeq C_{0}\left(T^{*} M\right) .
$$

(Here $B^{*} M$ is the unit ball bundle in the cotangent bundle.) 
The vertical mappings in the diagram are induced by quantizations. More precisely, the elements of the $K$-groups in the upper row are considered as difference elements for some elliptic symbols, and then one associates operators to the symbols. In more detail, $\chi$ corresponds to cone degenerate operators. The mappings $K_{c}^{*}\left(T^{*} M\right) \rightarrow K_{*}(M, \partial M)$ on the sides of the diagram are well known (e.g., see [2], [25]) and correspond to operators elliptic on the interior of the manifold (let us recall that in the case of odd $K$-homology groups one considers only self-adjoint elliptic operators). Such operators almost-commute with all functions vanishing on the boundary. All the vertical mappings, except possibly $\chi$, are isomorphisms (e.g., see [2]).

5. Let us compute the boundary mapping $\partial$. To this end we interpret the group $K_{c}^{1}\left(T^{*} M\right) \simeq K_{c}\left(T^{*} M \times \mathbb{R}\right)$ as the group generated by symbols $\sigma(x, \xi, \lambda)$ elliptic with parameter $\lambda \in \mathbb{R}$. Here the coordinates $x$ in a neighborhood of the boundary are separated into the tangential and normal $x=(y, t)$, the dual variables are denoted by $\xi=(\eta, p)$. Then the restriction of $\sigma(x, \xi, \lambda)$ to the boundary can be considered as the family of symbols on the boundary elliptic with parameters $(p, \lambda) \in \mathbb{R}^{2}$. Therefore, the index of this family is a number

$$
\text { ind } \sigma\left(y, 0,-i \frac{\partial}{\partial y}, p, \lambda\right) \in K_{c}\left(\mathbb{R}^{2}\right) \simeq \mathbb{Z} \text {. }
$$

This is well defined, since parameter dependent ellipticity implies that the operator family $\sigma(y, 0,-i \partial / \partial y, p, \lambda)$ is Fredholm everywhere and invertible for large values of the parameters (e.g., see [26]).

Lemma 1 The boundary $\partial[\sigma]$ of an element $[\sigma] \in K_{c}^{1}\left(T^{*} M\right)$ is expressed by the formula

$$
\partial[\sigma]=\operatorname{ind} \sigma\left(y, 0,-i \frac{\partial}{\partial y}, p, \lambda\right) \in K_{c}\left(\mathbb{R}^{2}\right) \simeq \mathbb{Z} .
$$

Proof. Consider a commutative diagram

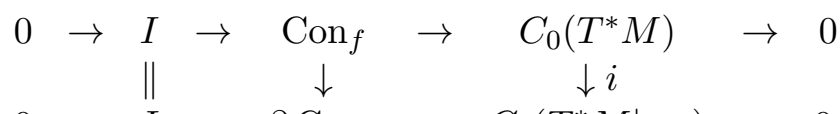

$$
\begin{aligned}
& 0 \rightarrow I \rightarrow \partial \mathrm{Con}_{f} \rightarrow C_{0}\left(\left.T^{*} M\right|_{\partial M}\right) \rightarrow 0,
\end{aligned}
$$

here $\partial \operatorname{Con}_{f}$ is the algebra

$C_{0}\left([0,1), \overline{\Psi_{p}(\partial M)}\right) \cap\left\{\sigma_{c}(0)-\right.$ operator of multiplication $\}=\operatorname{Con}\left(C(\partial M), \overline{\Psi_{p}(\partial M)}\right)$.

The diagram (24) and the naturality of the boundary mapping imply that the desired boundary mapping $\partial$ (it corresponds to the top row in (24)) is a composition

$$
\partial=\partial^{\prime} i_{*}
$$

of the restriction $i_{*}: K_{c}^{*}\left(T^{*} M\right) \rightarrow K_{c}^{*}\left(\left.T^{*} M\right|_{\partial M}\right)$ and the boundary mapping

$$
\partial^{\prime}: K_{c}^{*}\left(\left.T^{*} M\right|_{\partial M}\right) \rightarrow K_{*+1}(I)
$$

corresponding to the bottom row. 
We are interested in the boundary mapping $\partial^{\prime}$ defined on the odd $K$-group. Hence, the desired equality (23) follows from the fact that the boundary mapping from the odd group is actually the index (see [23]).

6. We can apply the 5-lemma to the diagram and thus prove the desired isomorphism $K_{0}\left(\mathrm{Con}_{f}\right) \simeq K_{0}(\mathcal{M})$, once we know the diagram is commutative. Therefore, the proof will be completed if we obtain the following lemma.

Lemma 2 The diagram (22) commutes.

Proof. A. Let us verify the commutativity of the square

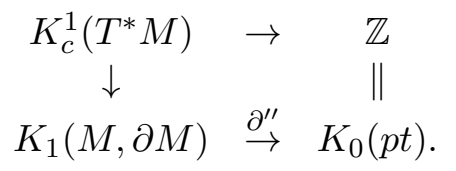

The boundary mapping $\partial^{\prime \prime}$ can be represented as the composition of the boundary mapping $K_{1}(M, \partial M) \rightarrow K_{0}(\partial M)$ and the index mapping $K_{0}(\partial M) \rightarrow K_{0}(p t)=\mathbb{Z}$, i.e. $\partial^{\prime \prime}$ coincides with the mapping in the upper row of the diagram (22) (see also [27]).

B. Commutativity of the square in the center of (22). The range of $\mathbb{Z} \rightarrow$ $K_{0}\left(\mathrm{Con}_{f}\right)$ is defined by elliptic operators of the form $1+\mathbf{G}$, where $\mathbf{G}$ is an operator with principal symbol equal to zero. We have to prove that such operators give Fredholm modules equivalent to a Fredholm module with a very simple module structure: the action of a function $f$ is determined by the product with the value $f(p t)$ at the cone vertex. This is accomplished in two steps:

1. (removal of the smooth part) without changing the element in $K$-homology, we can restrict the operator $1+\mathbf{G}$ to a neighborhood of the conical point (the original operator and its restriction are stably equivalent);

2. (homotopy of the module structure) in a neighborhood of the conical point the module structure $f(x), u(x) \mapsto f(x) u(x)$ is reduced by a homotopy to $f(x), u(x) \mapsto f(p t) u(x)$. The homotopy corresponds to the simplest rescaling: $f(x \varepsilon) u(x)$.

C. Commutativity of the right most square follows immediately from the definition of $K$-homology for noncompact spaces (i.e., $K$-theory for nonunital algebras), see 25$]$.

Thus, Theorem 1 is proved for the case, when there is only one singular point. For several conical points the ideal $I$ of operators with zero principal symbol is a direct sum of ideals $C_{0}\left(\mathbb{R}^{2}, \mathcal{K}\right)$ at each singular point, and we obtain

$$
K_{0}(I) \simeq \mathbb{Z}^{N}
$$

The scheme of the proof remains the same in this case and we shall not repeat it here. 


\subsection{Fibered manifolds}

In this subsection we prove Theorem 2 on the homotopy classification of elliptic operators on a fiber bundle.

1. As in the previous subsection we consider the classification for elliptic symbols. Furthermore, we classify continuous symbols. The inclusion of norm-closures of our algebras is denoted by

$$
f: C(X, \overline{\Psi(\Omega)}) \longrightarrow \overline{\Psi(Y, \pi)} / \mathcal{K} .
$$

The Calkin algebra of the closure $\overline{\Psi(Y, \pi)}$ is the subalgebra of compatible pairs of continuous symbols as in (91):

$$
\overline{\Psi(Y, \pi)} / \mathcal{K} \subset C\left(\overline{S^{*} Y \backslash \pi^{*} S^{*} X}\right) \oplus C\left(S^{*} X, \overline{\Psi(\Omega)}\right) .
$$

This follows from the equality of the norms of operators modulo compact terms, and the maximums of the absolute values of symbols (see [1] )

$$
\inf _{K \in \mathcal{K}}\|A+K\|_{L^{2}(Y) \rightarrow L^{2}(Y)}=\max \left(\max _{S^{*} Y}|\sigma(A)|, \max _{S^{*} X}\|\widehat{\sigma}(A)\|_{L^{2}(\Omega) \rightarrow L^{2}(\Omega)}\right) .
$$

2. We embed $\chi$ in a commutative diagram:

$$
\begin{array}{rcc}
K_{c}^{0}\left(T^{*} X\right) & \longrightarrow & K_{0}\left(\operatorname{Con}_{f}\right) \\
\simeq \searrow & \downarrow \chi \\
& K_{0}(X) .
\end{array}
$$

Here the horizontal mapping is induced by the embedding of the operators on the base $X$ in the set of operators on the fibration. This embedding is defined by realizing the usual finite-rank vector bundles on $X$ as the ranges of families of finite rank projections in the $L^{2}$-spaces on the fibers.

3. We will obtain the desired isomorphism property for $\chi$, if we prove that the horizontal mapping of the triangle is an isomorphism. This is proved in the following proposition.

Proposition 1 The inclusion of the ideal $I_{X} \subset \mathrm{Con}_{f}$ of elements with zero principal symbol induces the isomorphism of $K$-groups

$$
K_{0}\left(\operatorname{Con}_{f}\right) \simeq K_{0}\left(I_{X}\right) \simeq K_{c}^{0}\left(T^{*} X\right) .
$$

Proof. The quotient $\operatorname{Con}_{f} / I_{X} \simeq C_{0}\left(\overline{T^{*} M \backslash \pi^{*} T^{*} X}\right)$ corresponds to the product $[0, \infty) \times \partial\left(\overline{T^{*} Y \backslash \pi^{*} T^{*} X}\right)$, i.e., is contractible and has trivial $K$-groups. Thus, the short exact sequence of the pair $I_{X} \subset \mathrm{Con}_{f}$ induces the desired isomorphism of the $K$-groups of the algebra and its ideal.

The isomorphism $K_{0}\left(I_{X}\right) \simeq K_{c}^{0}\left(T^{*} X\right)$ follows from the fact that the ideal $I_{X}$ consists of functions on $T^{*} X$ with values in compact operators in the fibers. 


\subsection{Manifolds with fibered boundary}

In this subsection we prove Theorem 3 about the homotopy classification of elliptic operators on manifolds with fibered boundary.

1. The difference construction gives an isomorphism $\operatorname{Ell}(M, \pi) \simeq K_{0}(\mathrm{Con})$, where Con denotes the cone of the embedding:

$$
C(M) \longrightarrow \overline{\Psi(M, \pi)} / \mathcal{K} .
$$

Here $C(M)$ and $\overline{\Psi(M, \pi)}$ are the norm-closures of the smooth algebra of functions and the algebra of operators.

2. The groups Ell and $K$-will not change, if we replace the latter map by the monomorphism

$$
f: \widetilde{C}_{0}(M) \longrightarrow \overline{\Psi_{0}(M, \pi)} / \mathcal{K} .
$$

Here $\Psi_{0}(M, \pi) \subset \Psi(M, \pi)$ is the subalgebra of operators with symbols equal to zero on $\partial M$, and the algebra of "functions" $\widetilde{C}_{0}(M)$ is defined as:

$$
\widetilde{C}_{0}(M)=\left\{(u, v) \in C\left(M \backslash U_{\partial M}\right) \oplus C(X \times(0,1], \overline{\Psi(\Omega)}) \mid u_{t=1}=v_{t=1}\right\} .
$$

Here $t \in[0,1]$ is a normal coordinate in a neighborhood $U_{\partial M}$ of the boundary, $\{t=0\}$ is the equation of the boundary. This choice of the algebras corresponds to the class of operators introduced in Remark 2

3. We will prove the isomorphism for the $K$-groups $K_{0}\left(\mathrm{Con}_{f}\right)$ and $K_{0}(\mathcal{M})$.

4. The groups are embedded in the diagram:

$$
\begin{array}{ccccccccc}
K_{c}^{1}\left(T^{*} M\right) & \rightarrow & K_{0}\left(\mathcal{A}_{0}\right) & \rightarrow & K_{0}\left(\mathrm{Con}_{f}\right) & \rightarrow & K_{c}^{0}\left(T^{*} M\right) & \rightarrow & K_{1}\left(\mathcal{A}_{0}\right) \\
\chi_{M} \downarrow \simeq & & \chi_{X} \downarrow \simeq & & \chi_{\mathcal{M}} \downarrow & & \chi_{M} \downarrow \simeq & & \chi_{X} \downarrow \simeq \\
K_{1}(M \backslash \partial M) & \stackrel{\partial}{\rightarrow} K_{0}(X \times[0,1]) & \rightarrow & K_{0}(\mathcal{M}) & \rightarrow & K_{0}(M \backslash \partial M) & \stackrel{\partial}{\rightarrow} & K_{1}(X \times[0,1]) .
\end{array}
$$

Let us define this diagram. The bottom row here is the $K$-homology exact sequence of the pair $X \times[0,1] \subset \mathcal{M}$. The top row is induced by the exact sequence of algebras

$$
0 \longrightarrow \mathcal{A}_{0} \longrightarrow \operatorname{Con}_{f} \stackrel{j}{\longrightarrow} C_{0}\left(T^{*} M_{0}\right) \longrightarrow 0,
$$

here $j$ is the restriction mapping to the closed subset $M_{0}=M \backslash U_{\partial M}$, where we deal with the usual scalar symbols. The kernel of $j$ is an ideal in $\mathrm{Con}_{f}$ denoted by $\mathcal{A}_{0}$. One can see that this ideal is the mapping cone of the homomorphism:

$$
f_{(0,1)}: C_{0}(\partial M \times(0,1)) \rightarrow \overline{\Psi(\partial M \times(0,1), \pi)} / \mathcal{K} .
$$

In particular, according to Theorem 4 its $K$-group $K_{0}\left(\mathcal{A}_{0}\right) \simeq K_{0}(X)$ classifies elliptic operators corresponding to the fibration $\pi: \partial M \times[0,1] \rightarrow X \times[0,1]$ (see Subsection 2.2).

The vertical mappings in (27) - quantizations $\chi$ with lower indices - are defined in terms of elliptic operators: $\chi_{M}$ corresponds to operators on the interior of $M$, $\chi_{X}$ - to operators on the fibration $\pi$.

All the vertical mappings, except $\chi_{\mathcal{M}}$, are isomorphisms. Therefore, to prove that $\chi_{\mathcal{M}}$ is an isomorphism using the 5-lemma, it is enough to prove the commutativity of the diagram. 
5. Let us show that the left- and rightmost squares in (27) commute. The commutative diagram

$$
\begin{array}{cccccc}
\partial M \times[0,1] & \subset & M & \rightarrow & M / \partial M \\
\pi \downarrow & & \downarrow & & \| \\
X \times[0,1] & \subset & \mathcal{M} & \rightarrow & M / \partial M
\end{array}
$$

implies that the boundary mapping $\partial$ in (27) is a composition

$$
K_{*}(M, \partial M) \longrightarrow K_{*+1}(\partial M) \stackrel{\pi_{*}}{\longrightarrow} K_{*+1}(X)
$$

of the restriction to the boundary and the change of the module structure (direct image mapping $\pi_{*}$ ). Applying Poincaré isomorphism (see [3], 2]), we can pass to the topological $K$-groups, and rewrite (30) as

$$
K_{c}^{*}\left(T^{*} M\right) \longrightarrow K_{c}^{*}\left(\left.T^{*} M\right|_{\partial M}\right)=K_{c}^{*+1}\left(T^{*} \partial M\right) \stackrel{\pi_{!}}{\longrightarrow} K_{c}^{*+1}\left(T^{*} X\right),
$$

here $\pi_{!}$is the direct image mapping in $K$-theory induced by $\pi$.

It remains to compare this mapping with the boundary mapping $K_{c}^{*}\left(T^{*} M\right) \longrightarrow$ $K_{*+1}\left(\mathcal{A}_{0}\right)$ in the top row of (27). The latter mapping is also a composition

$$
K_{c}^{*}\left(T^{*} M\right) \stackrel{i_{*}}{\longrightarrow} K_{c}^{*}\left(\left.T^{*} M\right|_{\partial M}\right) \stackrel{\partial^{\prime}}{\longrightarrow} K_{*+1}\left(\mathcal{A}_{0}\right)
$$

of the restriction to the boundary $\partial M$ and the boundary mapping in $K$-theory (denoted by $\partial^{\prime}$ ) corresponding to the bottom row of the following diagram

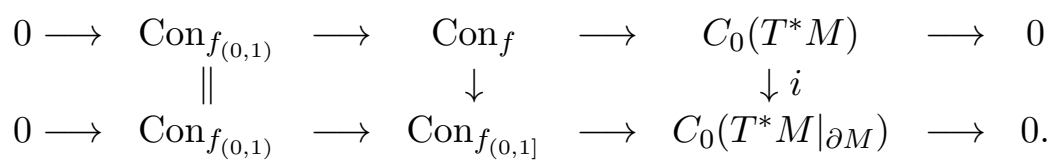

Here $f_{(0,1]}$ is the restriction of $f$ to $(0,1] \times \partial M$.

Let us compute $\partial^{\prime}$. To this end, we rewrite the sequence in terms of symbols on the boundary. By $\partial f: C(X, \overline{\Psi(\Omega)}) \rightarrow \overline{\Psi(\partial M, \pi)} / \mathcal{K}$ denote the inclusion of algebras associated with the fibered boundary.

The bottom row sequence in (31) can be represented in the form (21). Namely, it is obtained

$$
0 \rightarrow \mathcal{A}_{0}=\Sigma^{2} \operatorname{Con}_{\partial f} \rightarrow \Sigma \mathrm{Con}_{h} \rightarrow \Sigma C_{0}\left(T^{*} \partial M\right) \rightarrow 0
$$

as the suspension ${ }^{5}$ of the sequence of the form (21) induced by the homomorphism

$$
h: C_{0}\left(T^{*} \partial M\right) \rightarrow \operatorname{Con}_{\partial f} .
$$

The latter homomorphism is induced by the embedding of the continuous symbols in the class of discontinuous symbols.

Thus, according to (20) the boundary $\partial^{\prime}$ in $K$-theory

$$
K_{*}\left(C_{0}\left(T^{*} \partial M\right)\right) \stackrel{\partial^{\prime}}{\longrightarrow} K_{*}\left(\mathrm{Con}_{\partial f}\right)
$$

\footnotetext{
${ }^{5}$ The suspension common to all terms of the sequence uses the conormal variable.
} 
coincides with the induced mapping $h_{*}$.

Therefore, the commutativity of the extreme squares in (27) reduces to the commutativity of the following diagram

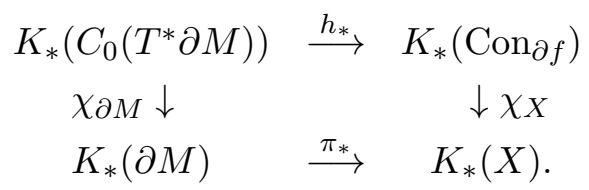

It is commutative, since $\partial M$ is closed and smooth.

6. The commutativity of the two squares in the middle of (27) is proved easily.

Applying the 5-lemma to (27) we end the proof of Theorem 3

\section{Applications and remarks}

Obstructions to Fredholm problems for elliptic operators. Let $\mathcal{M}$ be a manifold with edge $X$. Choose a diffeomorphism $\mathcal{M} \backslash X \simeq \stackrel{\circ}{M}$ of the smooth part and the interior of a smooth compact manifold $M$ with boundary. Let $\pi: \partial M \rightarrow X$ be the natural projection.

Let $D$ be an operator on $\mathcal{M}$ with elliptic interior principal symbol. As we know, this condition does not guarantee the Fredholm property of $D$. Then the following question naturally arises: can one make $D$ Fredholm by some modification of lower order terms of the operator?

It appears that the homotopy classification enables one to answer a slightly weaker version of this question, when instead of modifying lower order terms one can make stable homotopies of the principal symbol of the operator.

Corollary 1 Let $[D] \in K_{0}(\mathcal{M} \backslash X)$ be an element defined by an operator $D$ with elliptic interior principal symbol. Then this element can be lifted to an element in $K_{0}(\mathcal{M}) \simeq \operatorname{Ell}(\mathcal{M})$ defined by some operator elliptic on the entire space $\mathcal{M}$ if and only if

$$
\partial[D]=0,
$$

where $\partial: K_{0}(\mathcal{M} \backslash X) \longrightarrow K_{1}(X)$ is the boundary map of the exact sequence of the pair $X \subset \mathcal{M}$.

This readily follows from the exact sequence $\rightarrow K_{0}(\mathcal{M}) \rightarrow K_{0}(\mathcal{M} \backslash X) \stackrel{\partial}{\rightarrow} K_{1}(X) \rightarrow$.

In specific situations this formula gives many obstructions known previously.

1. For $X=\partial M$, i.e. when $\mathcal{M}=M$ is a manifold with boundary, the vanishing of $\partial[D] \in K_{1}(\partial M)$ is equivalent to the vanishing of the Atiyah-Bott [10] element $\left[\left.\sigma(D)\right|_{\partial T^{*} M}\right] \in K^{0}\left(\left.T^{*} M\right|_{\partial M}\right) \simeq K^{1}\left(T^{*} \partial M\right)$. The equivalence is given by Poincare duality $\left.K_{*}(\partial M) \simeq K^{*}\left(T^{*} \partial M\right)\right)$.

2. If $\mathcal{M}$ is a general manifold with edges, then the application of Poincare duality to $\partial[D]$ gives the element

$$
\pi_{!}\left[\left.\sigma(D)\right|_{\partial T^{*} M}\right] \in K^{1}\left(T^{*} X\right) .
$$

This formula for the obstruction was obtained in [17] using a different method. 
3. For isolated singularities ( $\operatorname{dim} X=0)$ the obstruction can be nontrivial only in the self-adjoint setting, i.e., for $[D] \in K_{1}(\mathcal{M} \backslash X)$. In this case the obstruction reduces to the so called deficiency indices, e.g. see [28], which are the obstructions to self-adjoint extensions of symmetric operators.

Let us finally note that the equality $\partial[D]=0$ can be effectively verified modulo torsion.

Proposition $2 \partial[D]=0 \in K_{1}(X) \otimes \mathbb{Q}$ if and only if

$$
\left\langle\left[\left.\sigma(D)\right|_{\partial T^{*} M}\right], \pi^{*} a\right\rangle=0
$$

for all elements $a \in K^{1}(X)$. Here

$$
\langle,\rangle: K^{1}\left(T^{*} \partial M\right) \times K^{1}(\partial M) \rightarrow K^{0}\left(T^{*} \partial M\right) \stackrel{\text { ind }}{\longrightarrow} \mathbb{Z}
$$

is the natural index pairing.

Proof. By the naturality of the boundary mapping and the index pairing we get:

$$
\langle\partial[D], a\rangle=\left\langle\pi_{*} \partial^{\prime}[D], a\right\rangle=\left\langle\partial^{\prime}[D], \pi^{*} a\right\rangle,
$$

where $\partial^{\prime}: K_{0}(\mathcal{M} \backslash X)=K_{0}(M \backslash \partial M) \rightarrow K_{1}(\partial M)$ is the boundary map for the pair $\partial M \subset M$.

By Poincare duality (33) is equal to $\left\langle\left[\left.\sigma(D)\right|_{\partial T^{*} M}\right], \pi^{*} a\right\rangle$. The equality $\langle\partial[D], a\rangle=$ $\left\langle\left[\left.\sigma(D)\right|_{\partial T^{*} M}\right], \pi^{*} a\right\rangle$ proves the desired statement, since the pairings are nondegenerate (Poincare duality).

$K$-groups of $C^{*}$-algebras of pseudodifferential operators. The problem of computing the $K$-groups of norm-closures of algebras of pseudodifferential operators on manifolds with singularities was considered in 29]. Let us show that the answer to this problem can be given using the homotopy classification.

First, we claim that the embedding $f: C(M) \rightarrow \overline{\Psi(\mathcal{M})} / \mathcal{K}$ induces a monomorphism in $K$-theory if $\mathcal{M}$ has no closed smooth components. This follows from the existence of a nonsingular vector field on $M$ : the vector field defines a left inverse $\overline{\Psi(\mathcal{M})} / \mathcal{K} \rightarrow C\left(S^{*} M\right) \rightarrow C(M)$ to $f$, and, hence, the 6 -term exact sequence splits:

$$
K_{i}(\overline{\Psi(\mathcal{M})} / \mathcal{K}) \simeq K^{i}(M) \oplus K_{i+1}\left(\operatorname{Con}_{f}\right) .
$$

Second, the exact sequence of the pair $\mathcal{K} \subset \overline{\Psi(\mathcal{M})}$ gives

$$
K_{0}(\overline{\Psi(\mathcal{M})}) \simeq K_{0}(\overline{\Psi(\mathcal{M})} / \mathcal{K}) \quad K_{1}(\overline{\Psi(\mathcal{M})}) \simeq \operatorname{ker}\left(K_{1}(\overline{\Psi(\mathcal{M})} / \mathcal{K}) \stackrel{\text { ind }}{\rightarrow} \mathbb{Z}\right)
$$

Combining these two results and the homotopy classification $K_{*}\left(\mathrm{Con}_{f}\right) \simeq K_{*}(\mathcal{M})$, we obtain

\section{Corollary 2}

$$
K_{i}(\overline{\Psi(\mathcal{M})}) \simeq K^{i}(M) \oplus \widetilde{K}_{i+1}(\mathcal{M}),
$$

where $\widetilde{K}_{*}$ is the reduced $K$-homology group. 
Classification, Poincare duality in $K$-theory. Relation to index theory. In terms of $K$-theory for $C^{*}$-algebras the homotopy classification gives an isomorphism

$$
K_{*}(\mathcal{A}) \stackrel{\simeq}{\rightarrow} K^{*}(C(\mathcal{M}))
$$

of $C^{*}$-algebraic $K$-groups of opposite variance. In $K$-theory such isomorphisms usually appear in pair with isomorphisms

$$
K^{*}(\mathcal{A}) \stackrel{\simeq}{\rightarrow} K_{*}(C(\mathcal{M}))
$$

of dual theories and one refers to $\mathcal{A}$ and $C(\mathcal{M})$ as Poincare dual algebras [30, 2].

Suppose for the moment that such a dual pair of isomorphisms exists. Denote by $x \in K^{0}(\mathcal{A})$ the preimage of the identity in $K_{0}(C(\mathcal{M}))=K^{0}(\mathcal{M})$. Then for an elliptic operator $D$ on $\mathcal{M}$ we obtain:

$$
\operatorname{ind} D=\langle[D], 1\rangle=\langle[\sigma(D)], x\rangle, \quad \text { where }[\sigma(D)] \in K_{0}(\mathcal{A}),
$$

by Poincare duality. The expression of the index in this form is important, because it contains only the principal symbol of $D$. If element $x$ is explicitly given then one can try to pass by Chern-Connes character to cyclic cohomology 30] and compute the corresponding class $\operatorname{ch} x \in H C^{*}(\mathcal{A})$ to obtain an index formula.

It would be very interesting to find a realizations of the element $x$ by some operator of geometric origin (some important steps in this direction can be found in [14 and 31]). Let us finally note that for a smooth manifold the element $x$ is given by the Dirac operator on $T^{*} M$. Thus, the problem is to realize the element $x$ by a Dirac like operator.

Let us note finally that the Poincare duality on manifolds with singularities is useful in the theory of index defects, see [32, where it is used to obtain index defect formulas. These topics for manifolds with singularities will be discussed elsewhere.

\section{Appendix. Analytic $K$-homology}

In this Appendix we recall for the readers convenience the basic definitions from the theory of analytic $K$-homology (see [33, 23, 34]), which are used throughout the paper. Let $X$ be a compact Hausdorf topological space.

Even Fredholm modules and the group $K_{0}(X)$.

Definition 4 An even Fredholm module over space $X$ is a pair $(F, H)$, consisting of a bounded operator

$$
F: H \rightarrow H,
$$

acting on the $\mathbb{Z}_{2}$-graded separable Hilbert space

$$
H=H_{0} \oplus H_{1},
$$

with components $H_{0,1}$ equipped with the structure of $*$-modules over the $C^{*}$-algebra $C(X)$. Denote the homomorphism defining the module structure by $\phi: C(X) \rightarrow$ 
$\mathcal{B}(H)$. We assume that the operator is odd relative to the grading, and for an arbitrary function $f \in C(X)$ we have

$$
\phi(f)\left(F-F^{*}\right) \in \mathcal{K}(H), \quad \phi(f)\left(F^{2}-1\right) \in \mathcal{K}(H), \quad[F, \phi(f)] \in \mathcal{K}(H),
$$

where $\mathcal{K}(H)$ is the ideal of compact operators in $H$.

The even $K$-homology group (with respect to direct sum) denoted by $K_{0}(X)$ is obtained from Fredholm modules, if we identify the modules by the equivalence relation - stable homotopy. ${ }^{6}$ Let us describe this equivalence relation.

Two Fredholm modules are isomorphic, if the corresponding $C(X)$-modules $H$ are isomorphic, and the operators $F$ transform one into another under the isomorphism of spaces. Two modules $\left(F_{1}, H\right)$ and $\left(F_{0}, H\right)$ are homotopic, if they can be connected by a family of modules $\left(F_{t}, H\right)$ such that the operator families $F_{t}$ and $\phi_{t}(f)$ (for fixed $f \in C(X)$ ) are strongly continuous. A module is trivial if it has each expression in (34) equal to zero (for any $f \in C(X)$ ). Finally, two modules are stably homotopic, if their direct sums with some trivial modules are homotopic.

Remark 5 The definition of the $K$-homology groups still makes sense if one replaces the commutative algebra $C(X)$ by much more general $C^{*}$-algebras. However, in this paper we use only the algebra $C(X)$ of continuous functions on a compact set or the algebra $C_{0}(M \backslash \partial M)$ of functions vanishing on the boundary of a smooth compact manifold $M$. The $K$-homology group of the latter nonunital algebra is called the relative $K$-homology group and denoted by $K_{0}(M, \partial M)$.

Odd Fredholm modules and the group $K_{1}$.

Definition 5 An odd Fredholm module over the space $X$ is a pair $(F, H)$. Here $F$ is a bounded operator

$$
F: H \rightarrow H,
$$

acting on a separable Hilbert space $H$, which is a $*$-module over the $C^{*}$-algebra $C(X)$ and for an arbitrary function $f \in C(X)$ one has

$$
\phi(f)\left(F-F^{*}\right) \in \mathcal{K}(H), \quad \phi(f)\left(F^{2}-1\right) \in \mathcal{K}(H), \quad[F, \phi(f)] \in \mathcal{K}(H) .
$$

Here $\phi$ is the module structure.

Thus, in contrast with the even case, the grading is not required. The set of stable homotopy classes of odd Fredholm modules over $X$ is denoted by $K_{1}(X)$.

Elliptic operators define elements of the groups $K_{*}$. The usual elliptic (pseudo)differential operators on a smooth closed manifold define elements in $K$ homology. Namely, if $D$ is an elliptic pseudodifferential operator in sections of some bundles $E$ and $F$ over a smooth closed manifold $M$, then it defines a bounded operator on $L^{2}$-spaces

$$
D^{\prime}=\left(P_{\text {ker } D}+D^{*} D\right)^{-1 / 2} D: L^{2}(M, E) \longrightarrow L^{2}(M, F) .
$$

${ }^{6}$ In [23], chapter VIII, one can find a number of other equivalence relations for Fredholm modules, which for a large class of $C^{*}$-algebras are equivalent to the relation we use in this paper. 
(Here $P_{\text {ker } D}$ is the orthogonal projection on the kernel of $D$.) Both $L^{2}$-spaces of sections are modules over the algebra $C(M)$. To construct a Fredholm module starting from $D^{\prime}$ one considers the matrix operator

$$
F=\left(\begin{array}{cc}
0 & D^{\prime *} \\
D^{\prime} & 0
\end{array}\right)
$$

as a self-adjoint operator in the naturally $\mathbb{Z}_{2}$-graded $C(M)$-module $H=L^{2}(M, E) \oplus$ $L^{2}(M, F)$. Operator $F$ is odd relative to the grading. The corresponding element in $K$-homology is denoted by

$$
\chi(D) \stackrel{\text { def }}{=}[F, H] \in K_{0}(M) .
$$

Odd Fredholm modules can be obtained from self-adjoint elliptic operators. Namely, if $A$ is an elliptic self-adjoint operator in sections of a vector bundle $E$ on a closed manifold, then the odd Fredholm module is defined using the operator

$$
A^{\prime}=\left(P_{\text {ker } A}+A^{2}\right)^{-1 / 2} A: L^{2}(M, E) \longrightarrow L^{2}(M, E) .
$$

The corresponding element in $K$-homology is denoted by

$$
\chi(A) \stackrel{\text { def }}{=}\left[A^{\prime}, H\right] \in K_{1}(M) .
$$

\section{References}

[1] M. F. Atiyah. Global theory of elliptic operators. In Proc. of the Int. Symposium on Functional Analysis, pages 21-30, Tokyo, 1969. University of Tokyo Press.

[2] G. Kasparov. Equivariant $K K$-theory and the Novikov conjecture. Inv. Math., 91(1):147-201, 1988.

[3] P. Baum and R. G. Douglas. K-homology and index theory. In R. Kadison, editor, Operator Algebras and Applications, number 38 in Proc. Symp. Pure Math, pages 117-173. American Mathematical Society, 1982.

[4] George W. Whitehead. Generalized homology theories. Trans. Amer. Math. Soc., 102:227-283, 1962.

[5] B.-W. Schulze. Pseudodifferential Operators on Manifolds with Singularities. North-Holland, Amsterdam, 1991.

[6] R. R. Mazzeo and R. B. Melrose. Pseudodifferential operators on manifolds with fibred boundaries. Asian J. Math., 4:833-866, 1998.

[7] V. Nistor. Singular integral operators on non-compact manifolds and analysis on polyhedral domains. arXiv: math.AP/0402322, 2004.

[8] V. Nazaikinskii, A. Savin, B.-W. Schulze, and B. Sternin. On the existence of elliptic problems on manifolds with edges. Russ. Acad. Sci. Doklady, 69(2):231$234,2004$. 
[9] V. Nistor. An index theorem for gauge-invariant families: The case of solvable groups. Acta Math. Hungarica, 99(2):155-183, 2003.

[10] M. F. Atiyah and R. Bott. The index problem for manifolds with boundary. In Bombay Colloquium on Differential Analysis, pages 175-186, Oxford, 1964. Oxford University Press.

[11] A. Savin and B. Sternin. Boundary Value Problems on Manifolds with Fibered Boundary. Chalmers Institute of Technology, Göteborg, December 2001. Preprint 75, available at arxiv.org/math.OA/0207179.

[12] M. F. Atiyah and I. M. Singer. The index of elliptic operators I. Ann. of Math., 87:484-530, 1968.

[13] V. Nazaikinskii, B.-W. Schulze, and B. Sternin. On the Homotopy Classification of Elliptic Operators on Manifolds with Singularities. Univ. Potsdam, Institut für Mathematik, Potsdam, Oktober 1999. Preprint N 99/21.

[14] C. Debord and J.-M. Lescure. $K$-duality for pseudomanifolds with isolated singularities. preprint math.OA/0212120, 2002.

[15] R. Melrose and P. Piazza. Analytic $K$-theory on manifolds with corners. Adv. in Math., 92(1):1-26, 1992.

[16] V. Nazaikinskii, A. Savin, B.-W. Schulze, and B. Sternin. On the Homotopy Classification of Elliptic Operators on Manifolds with Edges. Univ. Potsdam, Institut für Mathematik, Potsdam, 2004. Preprint N 2004/16.

[17] A. Yu. Savin and B. Yu. Sternin. Elliptic operators in even subspaces. Matem. sbornik, 190(8):125-160, 1999. English transl.: Sbornik: Mathematics 190, N 8 (1999), p. 1195-1228; arXiv: math/9907027.

[18] V. E. Nazaikinskii and B. Yu. Sternin. Operator algebras on manifolds with isolated singularities. Differential Equations, 39(1):92-104, 2003.

[19] Yu. Egorov and B.-W. Schulze. Pseudo-Differential Operators, Singularities, Applications. Birkhäuser, Boston, Basel, Berlin, 1997.

[20] M. Agranovich and M. Vishik. Elliptic problems with parameter and parabolic problems of general type. Uspekhi Mat. Nauk, 19(3):53-161, 1964. English transl.: Russ. Math. Surv. 19 (1964), N 3, p. 53-157.

[21] P. Haskell. Index theory of geometric Fredholm operators on varieties with isolated singularities. K-Theory, 1(5):457-466, 1987.

[22] B. A. Plamenevskii. On pseudodifferential operators with discontinuities in the symbols with respect to momenta and coordinates. Dokl. Akad. Nauk, 356(5):599-601, 1997.

[23] B. Blackadar. K-Theory for Operator Algebras. Number 5 in Mathematical Sciences Research Institute Publications. Cambridge University Press, 1998. Second edition.

[24] J. Cuntz and G. Skandalis. Mapping cones and exact sequences in $K K$-theory. J. Operator Theory, 15(1):163-180, 1986. 
[25] P. Baum, R. G. Douglas, and M. E. Taylor. Cycles and relative cycles in analytic K-homology. J. Differ. Geom., 30(3):761-804, 1989.

[26] M. A. Shubin. Pseudodifferential Operators and Spectral Theory. SpringerVerlag, Berlin-Heidelberg, 1985.

[27] R. Melrose and P. Piazza. Families of Dirac operators, boundaries and the b-calculus. J. of Diff. Geometry, 46(1):99-180, 1997.

[28] M. Lesch. Differential Operators of Fuchs Type, Conical Singularities, and Asymptotic Methods, volume 136 of Teubner-Texte zur Mathematik. B. G. Teubner Verlag, Stuttgart-Leipzig, 1997.

[29] R. Melrose and V. Nistor. $K$-theory of $\mathbf{C}^{*}$-algebras of $b$-pseudodifferential operators. Geom. Funct. Anal., 8(1):88-122, 1998.

[30] A. Connes. Noncommutative geometry. Academic Press Inc., San Diego, CA, 1994.

[31] V. Nazaikinskii, G. Rozenblioum, A. Savin, and B. Sternin. Guillemin Transform and Toeplitz Representations for Operators on Singular Manifolds. In G. Grubb B. Booss-Bavnbek and K. P. Wojciechowski, editors, Spectral Geometry of Manifolds with Boundary, Contemp. Math., 2005. (in print).

[32] A. Savin and B. Sternin. Index defects in the theory of nonlocal boundary value problems and the $\eta$-invariant. Sbornik:Mathematics, 195(9), 2004. Preliminary version at arXiv: math/0108107

[33] N. Higson and J. Roe. Analytic K-homology. Oxford University Press, Oxford, 2000 .

[34] Yu. P. Solovyov and E. V. Troitsky. $C^{*}$-algebras and elliptic operators in differential topology, volume 192 of Translations of Mathematical Monographs. American Mathematical Society, Providence, RI, 2001. 